\title{
INFLUENCE OF BOUND MAGNETIC POLARONS ON MAGNETIC AND ELECTRICAL PROPERTIES IN METALLIC $n-\mathrm{Cd}_{0.95} \mathrm{Mn}_{0.05} \mathrm{Se}^{*}$
}

\author{
P. Glód, M. Sawicki, T. Dietl, T. Skośkiewicz, A. Lenard, \\ AND W. PlesIeWICZ \\ Institute of Physics, Polish Academy of Sciences \\ Al. Lotników 32/46, 02-668 Warszawa, Poland
}

\begin{abstract}
Doping-induced contribution to the millikelvin magnetic susceptibility of $\mathrm{Cd}_{0.95} \mathrm{Mn}_{0.05} \mathrm{Se}: \mathrm{In}$ has been found to undergo a maximum at $n \approx 2 n_{c}$, and to vanish for $n \geq 8 n_{\mathrm{c}}$, where $n_{\mathrm{c}}$ is the electron concentration corresponding to the metal-insulator transition. This confirms the presence, also in the metallic phase, of bound magnetic polarons. Their slow dynamics may account for hysteresis visible in our magnetoresistance data.
\end{abstract}

PACS numbers: $71.30 .+h, 71.38 .+i, 75.20 . \mathrm{Hr}$

It is now believed [1] that a Hubbard-like conversion of the Fermi-liquid electrons into the local electron moments begins already on the metal side of the metal-to-insulator transition (MIT). In (diluted) magnetic semiconductors, localized electrons polarize via $s-d$ exchange interaction $M n$ spins within their localization radius, thereby creating ferromagnetic clouds of the aligned spins bound magnetic polarons (BMP). Therefore, the presence of BMP's via a strong spin-disorder scattering of itinerant electrons could determine low temperature transport properties of diluted magnetic semiconductors near MIT. Indeed, a strong and proportional to the magnetic susceptibility increase in the resistivity with decreasing temperature [2] as well as the appearance of a giant negative magnetoresistance in $n$-type $\mathrm{Cd}_{1-x} \mathrm{Mn}_{x}$ Se were observed [3].

We performed a systematic study of magnetic susceptibility $\chi$ in $\mathrm{Cd}_{0.95} \mathrm{Mn}_{0.05} \mathrm{Se}:$ In as a function of the electron concentration $n$, temperature $T$, and magnetic field $H$. The measurements were performed in a dilution refrigerator equipped with a modified mutual-inductance magnetometer, capable of detecting changes of $\chi$ below $10^{-6} \mathrm{emu}$ [4]. The a.c. magnetic field of the amplitude of $0.2-1 \mathrm{Oe}$ and frequency between 70 and $330 \mathrm{~Hz}$ was employed in the measurements.

*The work has been supported by grant No. 204669101 of the State Committee for Scientific Research (Republic of Poland). 


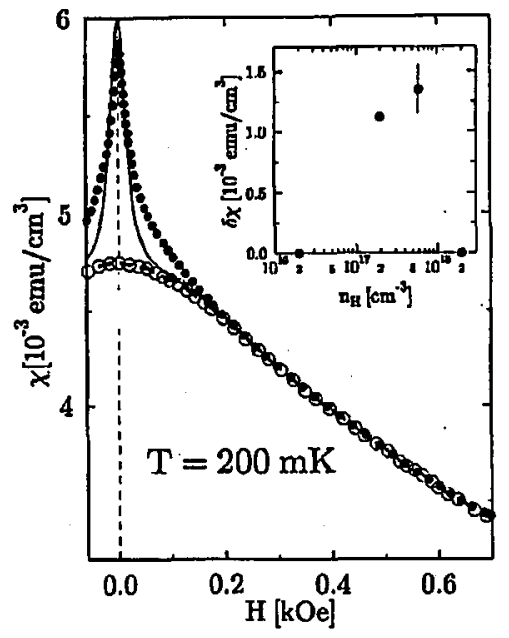

Fig. 1. Change of the magnetic susceptibility induced by the magnetic field for undoped (open circles) and In-doped (full circles) $\mathrm{Cd}_{0.95} \mathrm{Mn}_{0.05} \mathrm{Se}$. The inset shows the additional (relative to an undoped sample) contribution to $\chi$ for samples with different electron concentrations in $H=0$ Oe and $T=200 \mathrm{mK}$.

The field-induced changes of $\chi$ at $200 \mathrm{mK}$, well above the spin freezing temperature of $100 \mathrm{mK}[5,6]$, for samples with various $n$ up to $2 \times 10^{18} \mathrm{~cm}^{-3}$ are presented in Fig. 1. Since the critical electron concentration corresponding to the MIT is $n_{c}=(5 \pm 1) \times 10^{17} \mathrm{~cm}^{-3}$ in $n-\mathrm{Cd}_{0.95} \mathrm{Mn}_{0.05} \mathrm{Se}[2,3]$, the studied samples covered both sides of MIT. As shown, the field dependence of $\chi(H)$ in the undoped and the most strongly doped sample is similar. At the same time, $\chi$ of the remaining samples is strongly enhanced in the weak magnetic fields, $H \leq 200$ Oe. Such an additional contribution to $\chi$ may arise from BMP's, characterized by a large value of the total spin. This conjecture is strongly supported by our quantitative evaluation, shown by the solid line in Fig. 1. The calculation was performed according to the current theory of BMP [7], with the values of $\chi$ for the undoped sample taken as the background susceptibility (dashed line in Fig. 1). Thus, the BMP concentration $N_{\mathrm{p}}$ was the only adjustable parameter, and the solid line in Fig. 1 was computed for $N_{\mathrm{p}}=1 \times 10^{16} \mathrm{~cm}^{-3}$. We conclude that $N_{\mathrm{p}} \ll n$, i.e., only a small part of the electrons in the studied samples forms BMP's that are capable to respond to the weak a.c. magnetic field employed in our measurements. This may point to an antiferromagnetic coupling between the polarons and/or to the presence of local magnetic anisotropy.

The slow dynamics of BMP's suggested above is consistent with the results of our magnetoresistance measurements. As shown in Fig. 2, we observed pronounced hysteresis below $0.5 \mathrm{~K}$ while the magnetic field was swept through zero. The effect was found to depend strongly on the electron concentration, temperature and the sweep rate. Furthermore, the results shown reveal the presence of a noise-like structure in the intermediate range of the magnetic fields. This unusual behavior 


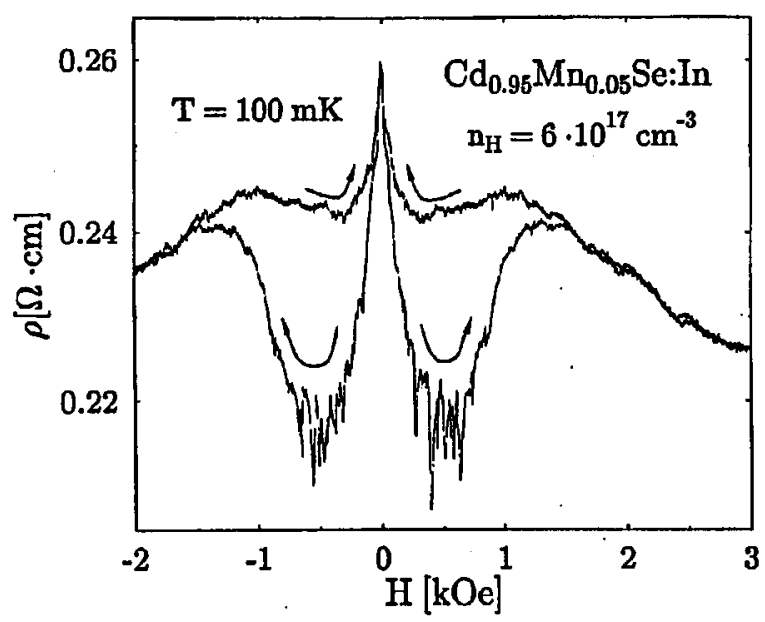

Fig. 2. Hysteresis of the magnetoresistance observed, while the magnetic field was swept through zero. The arrows indicate the direction of the sweeps. The sweep rate was $0.12 \mathrm{kOe} / \mathrm{min}$.

of the magnetoresistance may be a signature of macroscopic quantum tunneling of the individual BMP's. This interesting conjecture merits further studies.

\section{References}

[1] A. Gaymann, H.P. Geserich, H. v. Löhneysen, Phys. Rev. Lett. 71, 3681 (1993); P. Glód, T. Dietl, T. Fromherz, G. Bauer, I. Miotkowski, Phys. Rev. B 49, 7797 (1994), and references therein.

[2] M. Sawicki, T. Dietl, J. Kossut, J. Igalson, T. Wojtowicz, W. Plesiewicz, Phys. Rev. Lett. 56, 508 (1986); P. Głód, T. Dietl, M. Sawicki, I. Miotkowski, Physica B 194-196, 995 (1994).

[3] T. Dietl, L. Świerkowski, J. Jaroszyński, M. Sawicki, T. Wojtowicz, Phys. Scr. Vol. $T$ 14, 29 (1986).

[4] T. Lenard, W. Plesiewicz, M. Sawicki, T. Dietl, to be published in Cryogenics, 1994.

[5] M.A. Novak, O.G. Symko, D.J. Zheng, S. Oseroff, Physica B+C 126, 469 (1984); Phys. Rev. B 33, 6391 (1986).

[6] T. Dietl, M. Sawicki, T. Wojtowicz, J. Jaroszyński, W. Plesiewicz, L. Świerkowski, J. Kossut, in: Anderson Localization, Eds. T. Ando, H. Fukuyama, Springer, Berlin 1988 , p. 58.

[7] T. Dietl, J. Spalek, Phys. Rev. B 28, 1548 (1983); for recent measurements of BMP susceptibility, see T. Wojtowicz, S. Koleśnik, I. Miotkowski, J.K. Furdyna, Phys. Rev. Lett. 70, 2317 (1993); see also N.F. Oliveira Jr, S. Foner, Y. Shapira, T.B. Reed, Phys. Rev. B. 5, 2634 (1972). 